\title{
Active learning in optics for girls
}

\author{
R. Ali, I. Ashraf
}

R. Ali, I. Ashraf, "Active learning in optics for girls," Proc. SPIE 10452, 14th Conference on Education and Training in Optics and Photonics: ETOP 2017, 104520L (16 August 2017); doi: 10.1117/12.2269984

SPIE Event: 14th Conference on Education and Training in Optics and Photonics, ETOP 2017, 2017, Hangzhou, China 


\title{
ACTIVE LEARNING IN OPTICS FOR GIRLS
}

\author{
R. Ali and I. Ashraf \\ Department of Physics, Quaid-i-Azam University, Islamabad, Pakistan
}

\begin{abstract}
Active learning in Optics (ALO) is a self-funded program under the umbrella of the Abdus Salam International Centre for Theoretical Physics (ICTP) and Quaid-i-Azam University (QAU) to bring physical sciences to traditionally underserved Girls high schools and colleges in Pakistan. There is a significant gender disparity in physical Sciences in Pakistan. In Department of Physics at QAU, approximately 10 to $20 \%$ of total students were used to be females from past many decades, but now this percentage is increasing. To keep it up at same pace, we started ALO in January 2016 as a way to provide girls an enriching science experiences, in a very friendly atmosphere. We have organized many one-day activities, to support and encourage girls' students of government high schools and colleges to pursue careers in sciences. In this presentation we will describe our experience and lesson learned in these activities.
\end{abstract}

\section{KEYWORDS}

Optics education, optics outreach, hands on optics and photonics, active learning, inquiry based learning, predictions, observation, learning by doing, female education, laser learning

\section{MOTIVATION}

Pakistan is a developing country that is aiming to improve its economical growth and meet with the speed of the fast growing economies of the region. Pakistan is endeavoring to achieve development, such as eradicate of gender disparity at all levels, especially in education. Although the status of women has improved in recent years but gender imbalance remains persistent. Women are about $48.5 \%$ of Pakistan's total population. The talents of women are not completely exploited.

For achieving national development it is necessary to train young female students to the full at all levels of scientific and technological education. In order to address this issue we need to start from basics education in sciences for girls. The percentage of girls choosing physics as major hasn't improved much over the years. The time when we enrolled for our master program in late 80's, girls were ten to twenty percent of the total strength and after more than three decades this number is more or less the same, apart from couple of recent years at our university, number of girls enrolled in physics is almost equal to the boys. It seems there is something not right during early years of their education in high school and then at college level. Girls need to be able to self-identify that science education is not only important for them but also for society as a whole. This can be addressed by imparting them with a thorough knowledge of science in early years of school education. Good teachers and mothers can play an important role in a young girls' life, particularly in the Asian population. 


\section{Chart Title}

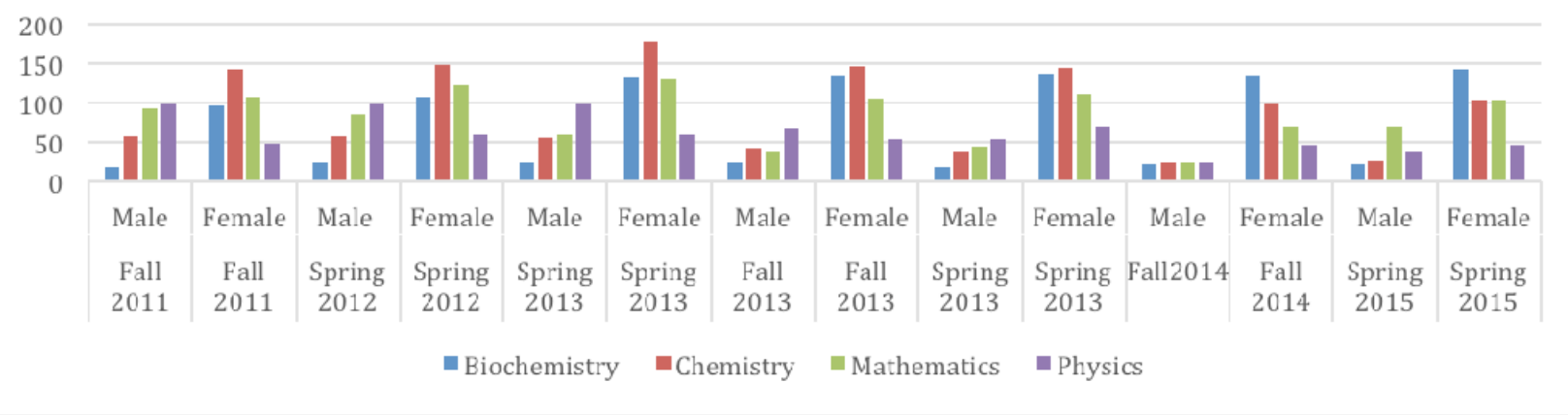

Figure 1. A bar-graph showing comparison of male and female students enrolled in four different departments of physical sciences from $2011-2015$.

\section{INTRODUCTION}

The United Nations declared 2015 as the International Year of Light (IYL2015) and light-based technologies. As a main result, the public interest is focused on both the achievements and the new frontiers of optics and photonics. This opens up new perspectives in the teaching and training of optics and photonics. But we should not forget that light touches upon much more than science. Given its central role in culture and art, an International Year of Light cannot only be for scientists and physicists, but also for all humanity. In order to take part in international year of light, an active learning in optics and photonics activity (ALOP 2015) was organized in Islamabad, Pakistan. Motivated by the success and good response rate of ALOP we decided to continue organizing hands on activities in optics for undergraduate students in general and young female students in particular. Keeping focus on:

1. To reach out and help government schools and colleges for girls

2. To help young girls to actively contribute to the learning process

3. To provide hands on and inquiry based training to school and college teachers

4. To reason in support of the significance of optics (science) to everyday life

5. To foster science as a force that can build up active citizens

6. To emphasize the relationship between science skills and future employability.

In order to achieve these goals we start with the fundamentals of light and lead the students to the critical role that light plays in our daily lives, to increase awareness of light- based technologies [1-4]. During the course of these activities we learnt following facts:

i) Education system in Pakistan is undergoing a major change: earlier students could get into the university for post-graduate study after ten years of school and four years of college education. According to the improved policy, that will be implemented from 2018, after twelve years of High School education students will get enrolled in four year Bachelors of Science program. While this will lead to new opportunities for physics graduates, this will surely add to the challenges they face in their classrooms. In order to make things understandable, class demonstrations should be an essential part at this level of teaching.

ii) Physics Education is neither part of a discipline nor a course in any University or College in Pakistan. As a consequence, professional training of Physics teaching is lacking; teachers learn teaching skills 
only by their personal experiences. In short they are doing a job that they never have been trained to do so.

iii) In Pakistan, Physics as a subject has been introduced during high school. Usually science teacher of that school teaches this subject, having no major in physics. The course work is not supported by experimental part as most government high school lack experimental facilities. Teachers try to cover the whole textbook without any conceptual details. Government schools for girls have much worse condition then boy's sections, due to less numbers of girls opting for sciences in high schools.

iv) During the first two years of college education, students opt for sciences if they plan to study medical or engineering. The ones who did not get admission in any professional colleges need to take twoscience subject to have Bachelor of Science degree. The choice can be biology with chemistry, or physics with mathematics, or physics with chemistry. Most common trend for girls doing Bachelor of Science is biology and chemistry.

v) Most girls' colleges don't support girls taking physics due to lack of good teachers in physics and laboratory facilities. Then the courses covered and examinations are not up to the mark as a result the students getting admissions in higher seats of learning lack understanding of foundational ideas in physics.

vi) During the course of our hands on activities we have found that practically no teacher training workshops are being arranged in their institutes to groom their teaching skills.

There can be many methodologies to develop interest in Sciences, particularly in Physics, and then one can lead them to field of optics, during their post- graduate level.

\section{LET'S SAY IT WITH LIGHT}

Our one-day optics activity is planned as follows: We start with a brief introduction of LIGHT in context with International Year of Light (IYL 2015) and discuss its importance and uses in our daily life.

We show different light sources, conventional light and LASER, and ask students what is the difference between these two and what is the importance of ordinary light and laser light in their respective lives. We make them understand that each light source has its own importance. We demonstrate that light travels in straight lines by illuminating the path of laser pointer with powder, based on scattering of light from powder particles.

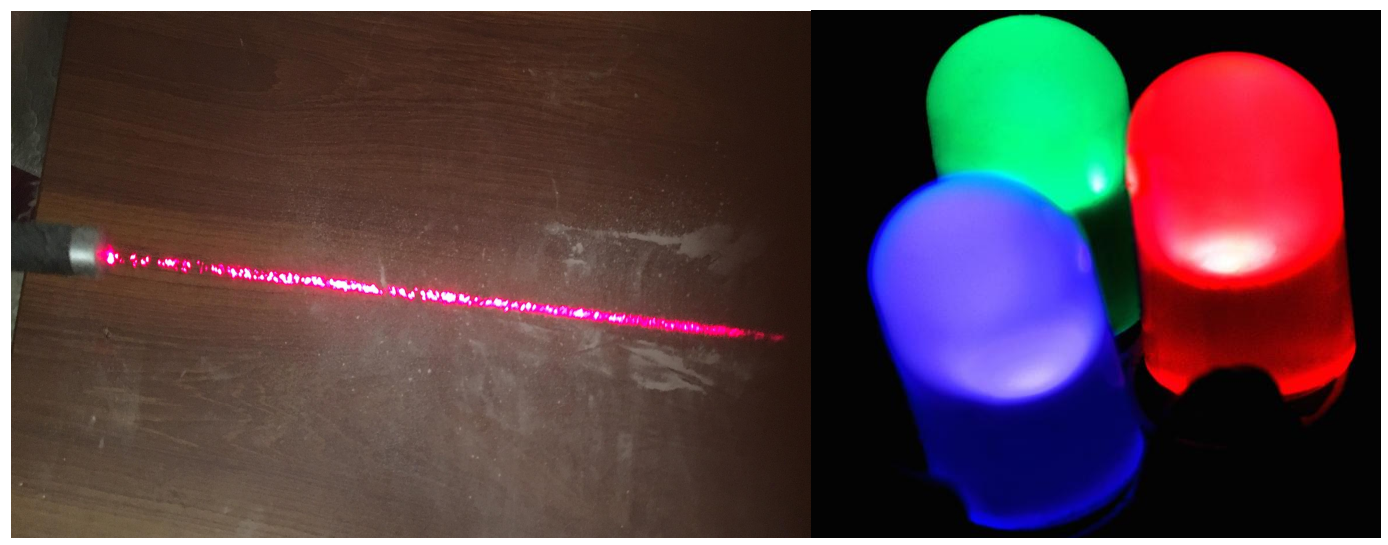

Figure 2. a) Diode laser light travelling in a straight line and visible due to scattering by the powder. b) Red, green and blue colors LED's. 


\subsection{Laws Of Geometrical Optics}

This part consists of verification of geometrical optics, like laws of reflection, refraction and total internal reflection. Students are given semi-circular dishes made of clear plastic and laser sources.

Semicircular dish is first filled with water and then few drops of milk are added to observe the path of the laser light. Then students are encouraged to make predictions that what will happen when ray of light passes from rare to denser medium or vice versa. At this point student can see reflection, refraction and transmission of light for different angles of incidence. This explains first two laws of geometrical optics without using any mathematical equations, like Snell's law of reflection. By changing the angle of incidence, students can observe the changing pattern of reflected, refracted and transmitted rays.
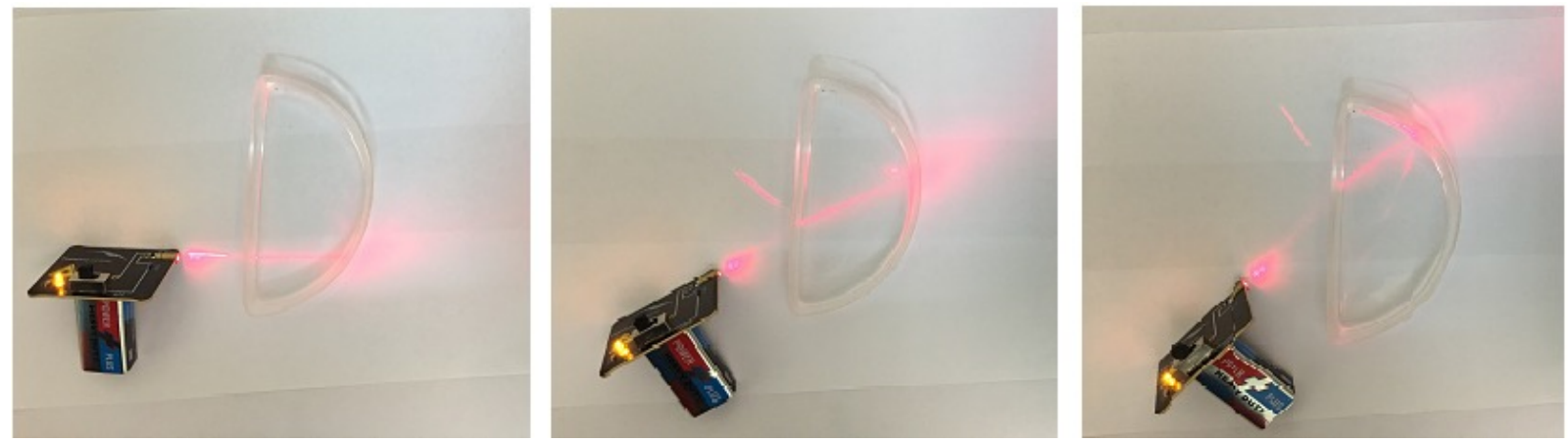

Figure 3. Reflection and refraction of light at various interfaces of a glass plate filled with water.

At certain critical angle, all of the light reflected back and there is no transmitted light. This situation is called total internal reflection. At this point students are given some basic knowledge of an optical fiber and light passing through a fiber optic cable is demonstrated. This helps them to understand how optical fibers work on the principle of totalinternal reflection and transmit light signals through long distances with out much loss.
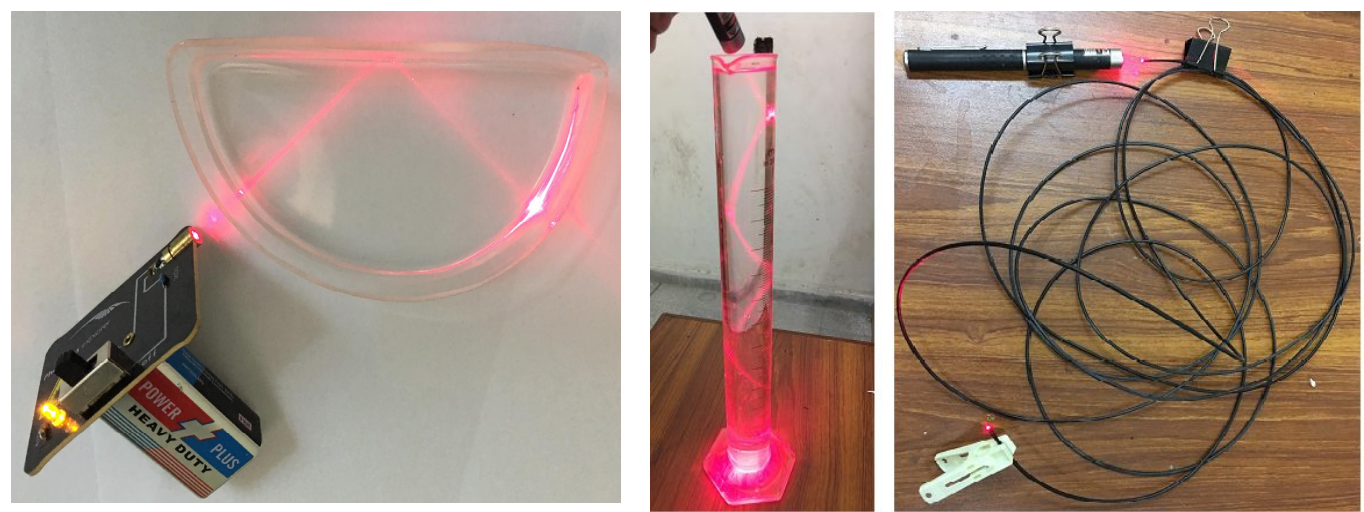

Figure 4. Total Internal Reflection of light from a) glass plate b) inside a water filled jar and c) through a fiber optics. 


\subsection{Interference And Diffraction}

This part of hands on activity helps students in understanding concepts of interference and diffraction. We found that often students mix up concept of diffraction pattern and interference fringes.

In physics, interference is a phenomenon in which waves superimpose to form dark and bright fringes. Interference pattern is observed with waves that are correlated or coherent with each other. This can be observed with all types of waves, for example, light, radio, acoustic, surface water waves or matter waves, but for the case of light it exhibits some very fascinating patterns.

The effects of diffraction are often seen in everyday life, but due to shorter wavelength of light it is difficult to demonstrate this phenomenon. One of the examples of diffraction are those that involve light; by using slits. For example, the closely spaced tracks on a Compact disc (CD) act as a diffraction grating to form the familiar rainbow pattern seen when looking at a disc. Using a simple compact disc (CD) we can do many wave optics experiments. Before doing experiment we stripe the metallic coating from CDs. Students can compare diffraction pattern of a CD (625 lines $/ \mathrm{mm})$ and commercially made grating $(1000$ lines $/ \mathrm{mm})$ present in photonics explorer kit. The striped CDs can be used to make a simple spectrometer, as shown below[7].

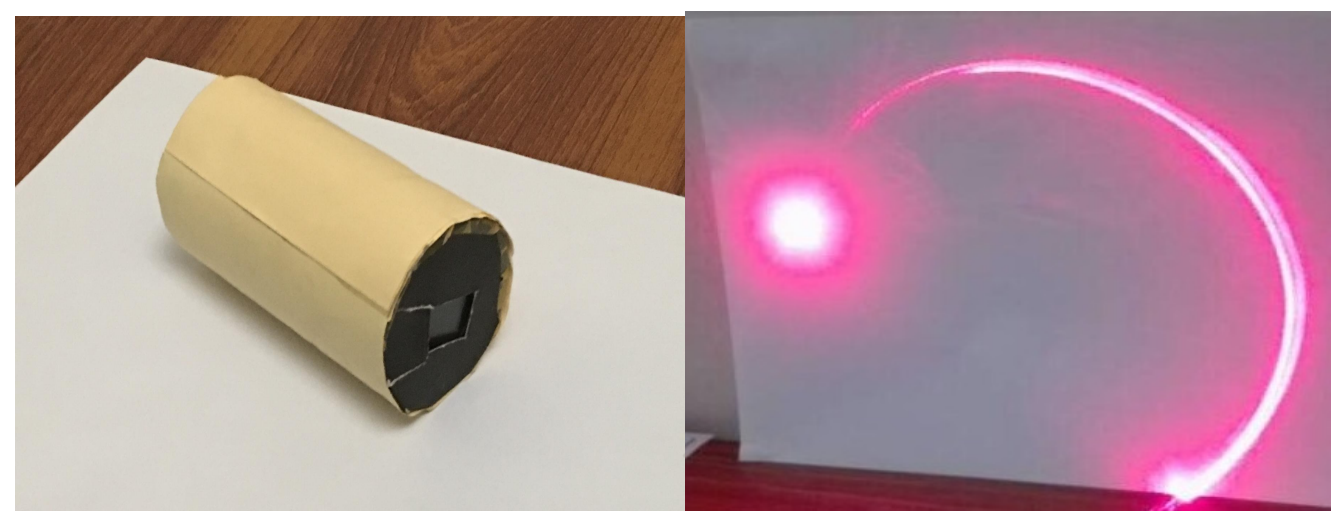

Figure 5. a) Hand-made spectrograph. b) Diffraction of light by a curved obstacle.

Diffraction phenomenon is used to separate different frequencies in different directions. As shorter frequency diffract with larger angle and vice versa, that is the basic concept of almost all spectrometers.

\subsection{Single And Double Slit}

Students are given a small black sheet-containing single slit, single bar, two double slits with different spacing's. They are asked to make prediction about the single slit and a bar, after that they verify by doing. Sometimes student are not able to relate Huygens principle with single slit diffraction pattern and need explanations. Interference from two slits makes clear sense to students, and they easily verify their concepts about it. Students are to think about making single and double slits using simple material, after some discussion we hand then some mirror stripes and sharp cutters to make slits. Then they compare results of commercially made and hand made slits [6]. 


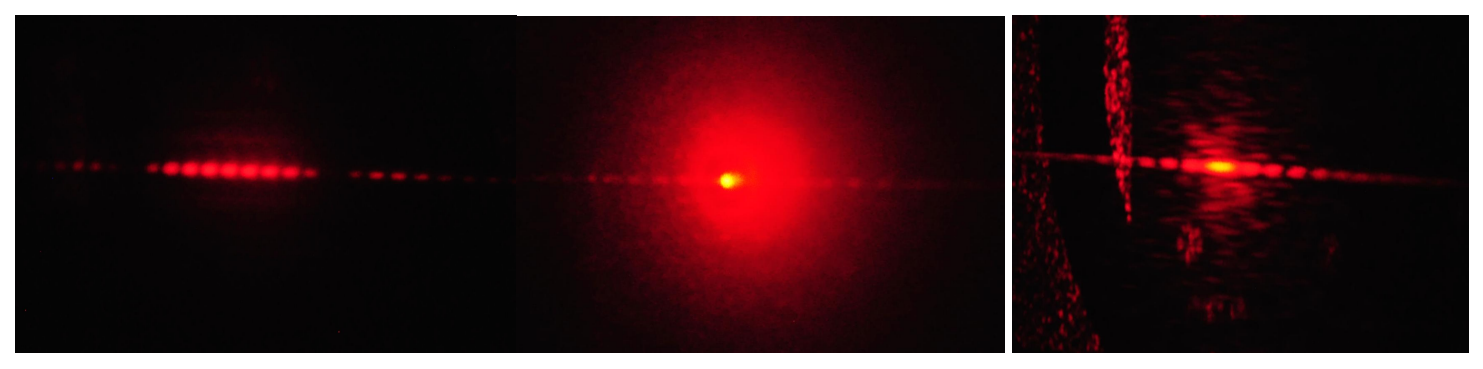

Figure 6. Diffraction pattern when laser light passes through a) Double slit b)through an obstacle and c) through a single slit.

\section{FUN WITH OPTICS}

\subsection{Optical Illusions}

We do certain optical illusions experiments and tricks during the course of these activities to show a fun with optics. That can be a demonstration of disappearing penny, burning candle in water, swapping the direction of arrows or magic words. We ask student how many of them have seen mirage effect in their respective lives. Why we see water on road in a hot summer day? We let them think and make predictions before watching these tricks that are mostly done by facilitators. After seeing these experiment they never forget about the role of reflection, refraction and refractive index [4].

\subsection{Shoebox's Projector}

With an understanding of hands on optics and photonics one can convert a shoebox into projector, with the help of magnifying glass and a smart phone. You can watch movies, video of your choice with friends and family.

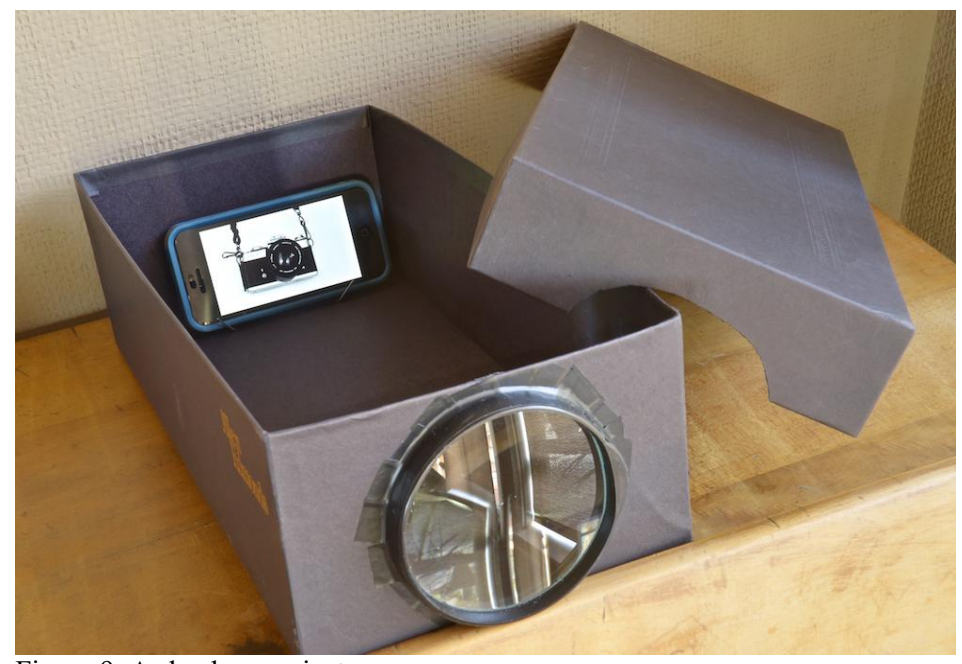

Figure 9. A shoebox projector 


\subsection{Swapping Colors With Optics}

All colors we see around us are not physical constants, but life is colorful. Just like beauty, colors exist literally in the eye of the beholder. We human have three different types of cell that recognize red, green and blue colors. It is fascinating that our brain focuses on just three, instead of being overwhelmed with di erent colors.
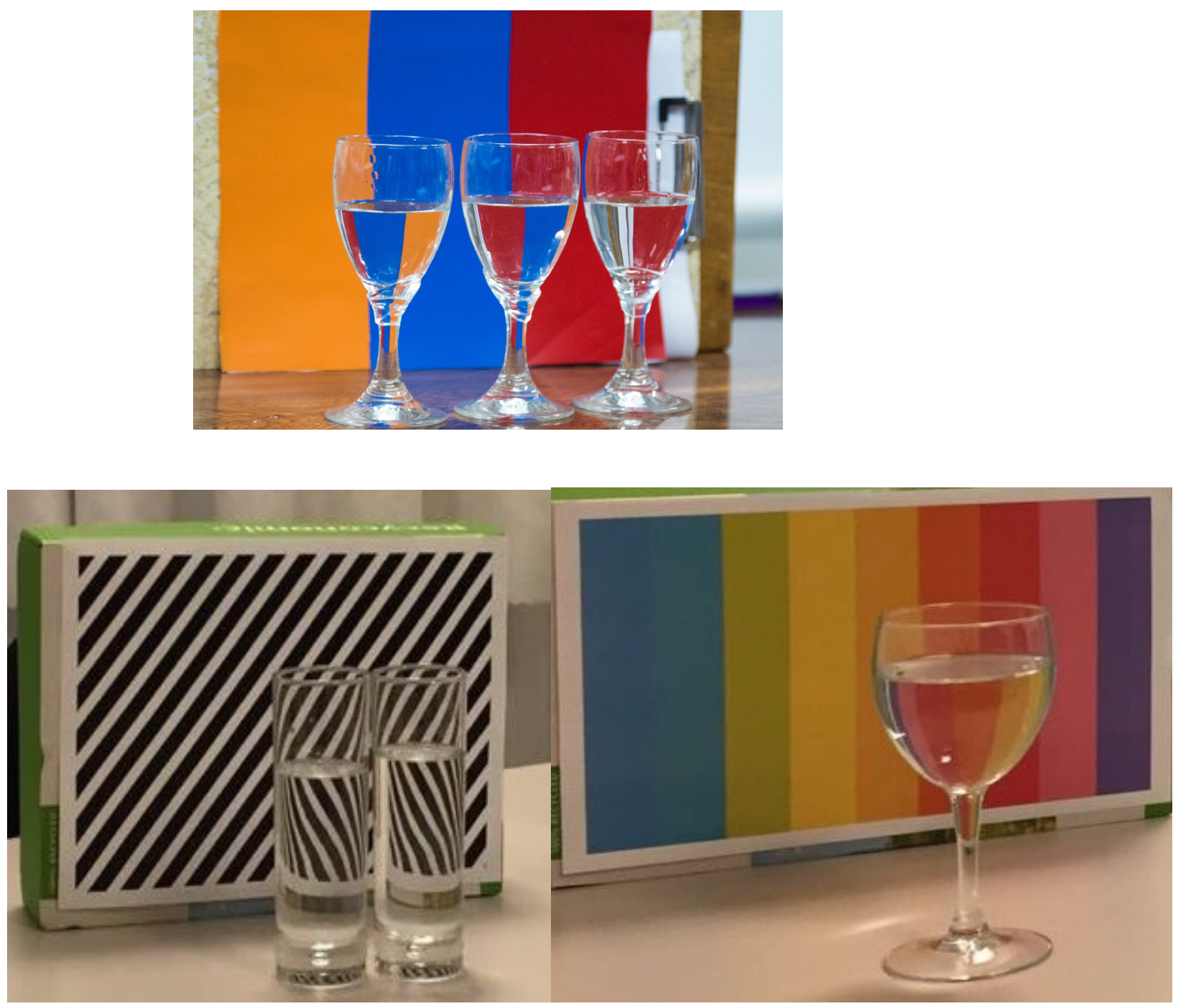

Figure 10 Change of position of colors and patterns through a glass filed with water. 


\section{CONCLUSIONS}

We have found the following aspects conclusive for the success of these hands on activities in optics and photonics.

- The timing of the hands activity should be at the end of each teaching year of college, and high school so that students can understand better the subject knowledge and scientific skills they have learnt throughout the year.

- A careful choice of topics should be made according to the theoretically background of students. In this way students can make connections to physics they already know and make significant progress in conceptual understanding of the subject by doing hands on activities.

- There should be an introductory session explaining aims of hands on activities before the start of the activity. In the introductory session, we get students to think and make predictions about the possible outcome of that particular activity. By doing hands on inquiry based activity they can verify their predictions.

Student should be encouraged to ask question and discuss with facilitators. The activities should not be too difficult in terms of underlying physics and should use at least some equipment students have seen at least. Rather then going into mathematical details about a concept, emphasis should on the qualitative reasons.

\section{FUTURE MOVES:}

The ALO group is planning to establish a regular calendar of outreach, hands on activities in Optics. In January 2016 we started with schools and colleges in Federal area of Pakistan, that is mainly consists of Islamabad (capital of Pakistan) and it suburbs. Having no physical boundaries with its twin city Rawalpindi (province of Punjab), our activities encircled schools and colleges of this area too. Now these activities are extended up to KPK provisional capital Peshawar. In coming year we would like to extend the limit of these activities to remote area of Pakistan and eventually make it a regional activity. We positively hope to take ALO (active learning in optics) to ALPS (active learning in physical sciences) that will also include Mechanics and Electromagnetism. As mentioned before ALO is so far a self-funded activity. We are also looking for any kind of support towards our outreach program, in terms of funds, materials and volunteers.

\section{ACKNOWLEDGEMENTS}

The authors wish to thank the Prof Joseph Niemela, for his interest, moral support and encouragement. We are also thankful for his kindness towards all material needed for these activities in the form of photonic explorer and APS kits. We would like to extend our gratitude to all our supporters, ICTP and QAU.

\section{References}

1. Meltzer D. E. and Thornton R.K. "Resource Letter ALIP-1: Active-Learning Instruction in Physics' Am. J. of Physics 80, 478 (2012)

2. Talisayon, V.M. "Physics teaching in developing countries Physics Education" 198419105 (1984).

3. Curticapean, D., "University for Children - The Magic of Light", Proceedings: Eleventh International Topical Meeting on Education and Training in Optics and Photonics, St. Asaph, North Wales, United Kingdom (5-7 June 2009)

4. Curticapean, D., "Magic of Light", Proc. SPIE 7783, Optics Education and Outreach, 77830I (31 August 2010); doi: $10.1117 / 12.862847,(2010)$.

5. Karamustafaoglu, O "Active learning strategies in physics teaching" Energy Education Science and Technology Part B: Social and Educational Studies 127 (2009).

6. Aviani, I and Erjavec, B. "An easy method to show the diffraction of light" Phys. Educ. 46134 (2011) and references therein

7. Westra M.T. “A fresh look at light: build your own spectrometer” Sci. School 430 (2007) 